\title{
Source Extraction in Bandwidth Constrained Wireless Sensor Networks
}

\author{
Hongbin Chen, Chi K. Tse, Fellow, IEEE, and Jiuchao Feng, Member, IEEE
}

\begin{abstract}
Source extraction was traditionally done by sensor arrays. Recently, sensor networks have been considered as promising candidates for extraction of multiple sources. In a sensor network, each sensor observes an instantaneous linear mixture of the sources and their observations are corrupted by additive white Gaussian noise. Two sensor network models are adopted. The first one is cluster based, in which a sensor acts as cluster head and performs local extraction of the sources based on its own observation and the received quantized data from the cluster members. Then, the extracted signal is quantized and the quantized data are sent to the sink while the sink performs global extraction of the sources. The other one is cluster free, in which data collected by the sensors are quantized and sent to the sink directly. Then, the sink performs global extraction of the sources. The proposed schemes are evaluated against the benchmarking case where the sensor observations are undistorted.
\end{abstract}

Index Terms-Blind source extraction, distributed estimation, wireless sensor network.

\section{INTRODUCTION}

W IRELESS sensor network is an emerging platform for application-oriented information retrieval tasks. In a wireless sensor network, small sensors are densely distributed in a sensing field and are connected by wireless links. These sensors have limited capability of sensing, data processing, radio communication, and are battery-powered. Through coordination of sensors, a sensor network can accomplish some complex tasks [1]. When designing a sensor network for practical use, several inherent constraints must be considered including limited available energy and limited communication bandwidth of sensors, noise corruption of sensor observations and distortion of wireless channels.

Recently, a great number of estimation problems in wireless sensor networks have been studied [2]-[8]. However, to our knowledge, little work has been devoted to the problem of source separation/extraction in wireless sensor networks. Source separation as a fundamental problem in signal processing has been pursued for more than two decades under the context of sensor arrays. Source extraction, which requires

Manuscript received January 11, 2008. First published June 10, 2008; current version published September 12, 2008. This work was supported by National Natural Science Foundation of China under Grant 60572025 and by the Hong Kong Polytechnic University under Grant G-YF51. This paper was recommended by Associate Editor S. Callegari.

H. Chen is with the Department of Electronic and Information Engineering, Hong Kong Polytechnic University, Hong Kong, and also with the School of Electronic and Information Engineering, South China University of Technology, Guangzhou 510641, China.

C. K. Tse is with the Department of Electronic and Information Engineering, Hong Kong Polytechnic University, Hong Kong.

J. Feng is with the School of Electronic and Information Engineering, South China University of Technology, Guangzhou 510641, China.

Digital Object Identifier 10.1109/TCSII.2008.924373 weak solvability conditions than source separation, seems easier to tackle [9].

In this paper, the problem of source extraction in bandwidth constrained wireless sensor networks is studied. Two sensor network models are adopted and simple schemes are proposed for extracting multiple sources in a sensing field. The main contribution of this work is to extend the study of the source extraction problem from sensor arrays to wireless sensor networks and to investigate how the unique characteristics of wireless sensor networks would affect the performance of source extraction. Two unique characteristics are considered: 1) Because of the bandwidth constraint, sensor observations should be quantized before transmission, thus quantization noise is introduced in the mixing model; 2) As source extraction is performed in a wireless network, sensor observations would be corrupted by noise and distorted by wireless channels during the transmissions. Specifically, we focus on the effect of the first characteristic in this paper.

The use of sensor networks has a lot of advantages over the use of sensor arrays, e.g., ease of deployment and elimination of manned data collection. These advantages come from the fact that in a sensor network, small sensors can be dropped down by air into a sensing field and self-organize into a network while in a sensor array, large sensors are usually deployed manually and they function individually.

\section{BENCHMARKING SOURCE EXTRACTION}

Source separation/extraction was traditionally done by sensor arrays. Under this circumstance, sensor observations are perfectly gathered in a fusion center which performs source separation/extraction. Without distortion of sensor observations, the performance is expected to be superior and will be used as a benchmark for evaluating our proposed schemes.

\section{A. Mixing Model}

Consider a scenario where $J$ sources in a sensing field are observed by $N$ sensors. The observation of sensor $n$ is assumed to be a noisy instantaneous linear mixture of the sources and is represented by

$$
y_{n}(t)=\sum_{j=1}^{J} a_{n j} s_{j}(t)+w_{n}(t), \quad n=1, \ldots, N
$$

where $y_{n}$ and $s_{j}$ are the $n$th observation and the $j$ th source, respectively; $w_{n}$ is the $n$th AWGN which is uncorrelated with $w_{m}(m \neq n)$ and is also uncorrelated with $s_{j} ; a_{n j}$ is the unknown mixing coefficient; $t$ denotes the discrete time $(t=0, \ldots, L-1)$. More complicated mixing cases are convolutive mixing and nonlinear mixing, which will not be discussed here. 
Let us define $\boldsymbol{Y}=\left[y_{1}, \ldots, y_{N}\right]^{T}, \boldsymbol{S}=\left[s_{1}, \ldots, s_{J}\right]^{T}, \boldsymbol{V}=$ $\left[w_{1}, \ldots, w_{N}\right]^{T}$, and $\mathbf{A}=\left[a_{n j}\right]$. Then, (1) converges to a vector model $\boldsymbol{Y}=\mathbf{A} \boldsymbol{S}+\boldsymbol{V}$.

Without loss of generality, the following assumptions are made.

A1: The sources are stationary, ${ }^{1}$ non-Gaussian, mutually independent, of zero-mean, and have unit variance.

A2: $J$ is known a priori and $N>J$.

A3: The mixing matrix $\mathbf{A}$ has full column rank.

Under the above assumptions, one can seek a separation matrix to recover the sources simultaneously or an extraction vector to recover the sources one by one, using some algorithm derived from a contrast function, which can handle inherent permutation and amplitude ambiguities.

\section{B. Source Extraction Algorithm}

Many robust algorithms have been proposed for source extraction. A fast fixed-point algorithm [10] is chosen here because of its simplicity and fast convergence. ${ }^{2}$ In each round, it can extract one independent non-Gaussian source from the mixtures regardless of the probability distributions of the sources. Before source extraction, to reduce the effect of noise, $Y$ is pre-whitened [11] by the following steps.

- Estimate the sample covariance $\hat{\mathbf{R}}(0)$ from $L$ samples of $\boldsymbol{Y}$.

- Take an eigen-decomposition of $\hat{\mathbf{R}}(0)$. Denote the $J$ largest eigenvalues of $\hat{\mathbf{R}}(0)$ by $\lambda_{1}, \ldots, \lambda_{J}$ and the corresponding eigenvectors by $\boldsymbol{h}_{1}, \ldots, \boldsymbol{h}_{J}$.

- Estimate the noise variance which is the average of the $N-J$ smallest eigenvalues of $\hat{\mathbf{R}}(0)$ and denote it by $\hat{\sigma}^{2}$. Then, $\mathbf{P}=\left[\left(\lambda_{1}-\hat{\sigma}^{2}\right)^{-1 / 2} \boldsymbol{h}_{1}, \ldots,\left(\lambda_{J}-\hat{\sigma}^{2}\right)^{-1 / 2} \boldsymbol{h}_{J}\right]^{T}$.

The whitened vector is $\boldsymbol{X}=\mathbf{P} \boldsymbol{Y}$. Then, the fast fixed-point algorithm is implemented as follows:

- Choose a random initial vector $w(0)$ of norm 1 . Set $\tau=1$.

- Start iteration: $\boldsymbol{w}(\tau)=E\left\{\boldsymbol{X}\left(\boldsymbol{w}(\tau-1)^{T} \boldsymbol{X}\right)^{3}\right\}-3 \boldsymbol{w}(\tau-1)$, where $E(\cdot)$ is the expectation operator and is numerically calculated by the average of samples.

- Divide $\boldsymbol{w}(\tau)$ by its norm.

- If $\left|\boldsymbol{w}(\tau)^{T} \boldsymbol{w}(\tau-1)\right|$ is not close enough to 1 , let $\tau=\tau+1$, and go back to iterate. Otherwise, stop iteration and output the vector $\boldsymbol{w}(\tau)$. It is known that $\boldsymbol{w}(\tau)^{T} \boldsymbol{X}$ equals one of the sources.

\section{Source EXtraction In SENSOR Networks}

In sensor networks, the usual information retrieval task is to extract one source in each round at a remote sink. Simultaneous extraction of multiple sources is normally not considered. Nonetheless, multiple sources extraction can be realized by adding an orthogonalizing projection operation in the fast fixed-point algorithm. Obviously, how to achieve this task depends on the kind of sensor network being used.

\section{A. Schemes}

Many sensor network models have been proposed in the literature. Two of them are adopted here, namely, cluster-based and

\footnotetext{
${ }^{1}$ Nonstationary sources like speech may also be separated/extracted.

${ }^{2}$ Other source extraction algorithms may also be applicable.
}

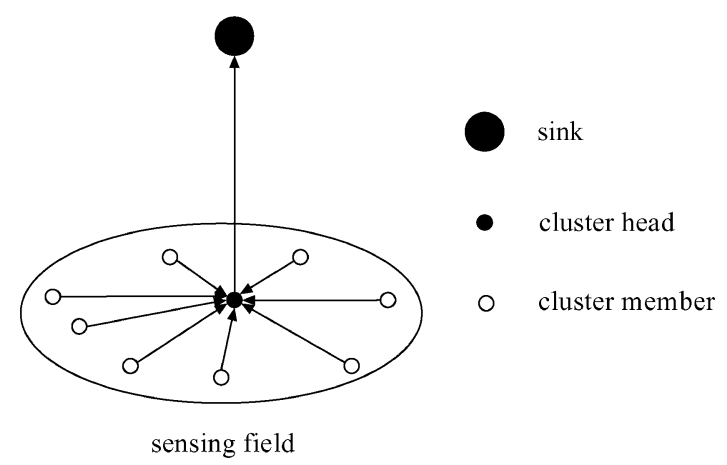

Fig. 1. Cluster-based sensor network.

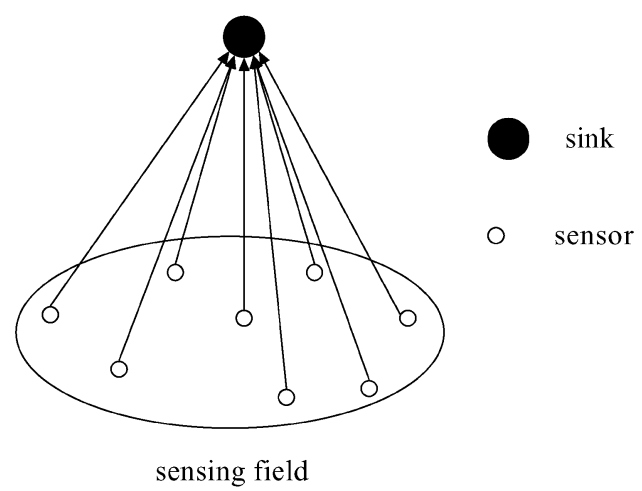

Fig. 2. Cluster-free sensor network.

cluster-free. Fig. 1 shows a cluster-based sensor network. In this sensor network, the source extraction process is divided into two stages. In the first stage, the cluster head performs local extraction of the sources based on its own observation and the received quantized data from the cluster members. In the second stage, the cluster head quantizes each extracted signal and sends the quantized data to the sink. Finally, the sink performs global extraction of the sources. A cluster-free sensor network is shown in Fig. 2. In this sensor network, each sensor quantizes its observation and sends the quantized data to the sink. Then, the sink performs global extraction of the sources. The basic idea of the proposed schemes is to reconstruct the original mixtures at the cluster head or the sink.

To facilitate the execution of the information retrieval task, the following conditions are assumed.

A4: The available energy and communication bandwidth of all sensors are limited. However, the cluster head has more resources than the cluster members in the network. Moreover, the sink has sufficient energy and communication bandwidth.

A5: Transmissions from the cluster members to the cluster head in the cluster-based sensor network or from the sensors to the sink in the cluster-free sensor network are through orthogonal channels so that interference can be ignored.

A6: The size of the sensor network considered is not too large that transmissions from/to sensors can be synchronized. 


\section{B. Algorithms}

The algorithms for source extraction in the sensor networks are elaborated in this subsection, including quantization of sensor observations, local extraction and global extraction.

1) Quantization of Sensor Observations: The probability distributions of the sources are not specified, so the uniform quantization method is employed to convert the sensor observations into binary sequences. Suppose the sensor observations are bounded over the interval $[-W, W]$. The quantization bit budget for sensor $i$ is $M_{i}(1 \leq i \leq N)$. First, $y_{i}$ is normalized to the range $[0,1]$ by a linear transformation

$$
\tilde{y}_{i}=\left(W+y_{i}\right) / 2 W \text {. }
$$

Then, local independent quantizers $Q_{i}: \tilde{y}_{i} \mapsto F_{i}\left(\tilde{y}_{i}, M_{i}\right)$ are designed and $\tilde{y}_{i}$ is quantized sample by sample, where $F_{i}\left(\tilde{y}_{i}, M_{i}\right)$ is a discrete message of $M_{i}$ bits and can be represented by

$$
\begin{aligned}
\left.\left(\tilde{y}_{i}-q_{i}\right)\right|_{t} & =\sum_{k=1}^{M_{i}} b_{k} 2^{-k}, \quad b_{k}=0,1 \\
F_{i}\left(\tilde{y}_{i}, M_{i}\right) & =b_{1} \cdots b_{M_{i}}
\end{aligned}
$$

where $q_{i}$ is the $i$ th quantization noise and the samples of $\tilde{y}_{i}$ are indexed by $t$. Note that the observation of the cluster head need not be quantized in the case of the cluster-based sensor network.

2) Local Extraction: In the case of the cluster-free sensor network, local extraction is not necessary. Moreover, in the case of the cluster-based sensor network, suppose the cluster members send the quantized data to the cluster head bit by bit without distortion. Then, the cluster head (denoted by sensor $\phi$ ) will receive a total of $N-1$ quantized messages which are denoted by

$$
z_{i, k}=F_{i}\left(\tilde{y}_{i}, M_{i}\right)_{k}, \quad k=1, \ldots, M_{i} .
$$

The original mixtures $y_{i}$ are reconstructed at sensor $\phi$ and are denoted by $\tilde{z}_{i}$, where

$$
\tilde{z}_{i}=2 W \sum_{k=1}^{M_{i}} z_{i, k} 2^{-k}-W .
$$

After reconstruction, sensor $\phi$ performs local extraction of the sources based on $y_{\phi}$ and the reconstructed mixtures $\tilde{z}_{i}$ (distorted, $\left.\tilde{z}_{i} \approx y_{i}\right)$. Though the mixtures are distorted, their waveforms retain many original features, thus making source extraction possible [12]. Let $\boldsymbol{Z}=\left[y_{\phi}, \tilde{z}_{1}, \ldots, \tilde{z}_{N}\right]^{T}$. Because the quantization noises are not zero-mean (uniformly distributed [13]), $Z$ is centered and pre-whitened before source extraction. The pre-whitening process is the same as before. After prewhitening, the fast fixed-point algorithm is used to extract the sources.

From 1-(6), the new mixing model is known to be $Z=$ $\mathbf{A} \boldsymbol{S}+\boldsymbol{U}$, where $\boldsymbol{U}=\left[w_{\beta}, w_{1}-2 W q_{1}, \ldots, w_{N}-2 W q_{N}\right]^{T}$. It is recognized from this model that quantization of sensor observations transfers the mixing model from noiseless to noisy or from noisy to noisier.

3) Global Extraction: In the case of the cluster-based sensor network, the cluster head quantizes each extracted signal and sends the quantized data to the sink. The locally extracted signals are denoted by $\hat{s}_{j}, j=1, \ldots, J$. The range of $\hat{s}_{j}$ is normalized to $[-1,1]$ and the normalized $\hat{s}_{j}$ is quantized sample by sample with 8-bit PCM encoding [14]. Then, sensor $\phi$ sends the quantized data bit by bit to the sink. Suppose all bits are correctly received by the sink. PCM decoding is then done based on the received bits which leads to a globally extracted signal. The globally extracted signal corresponding to the $j$ th source is denoted by $\gamma_{j}$.

In the case of the cluster-free sensor network, each sensor quantizes its observation and sends the quantized data to the sink, respectively. The bits received by the sink originating from sensor $i$ are

$$
\Gamma_{i, k}=F_{i}\left(\tilde{y}_{i}, M_{i}\right)_{k}, \quad k=1, \ldots, M_{i} .
$$

The original mixtures $y_{i}$ are reconstructed at the sink and are denoted by $\tilde{z}_{i}$, where

$$
\tilde{z}_{i}=2 W \sum_{k=1}^{M_{i}} \Gamma_{i, k} 2^{-k}-W .
$$

Then, the sink performs global extraction of the sources based on the reconstructed mixtures $\tilde{z}_{i}$. In this sensor network, the new mixing model is $\boldsymbol{Z}=\mathbf{A} \boldsymbol{S}+\tilde{\boldsymbol{U}}$, where $\tilde{\boldsymbol{U}}=\left[w_{1}-\right.$ $\left.2 W q_{1}, \ldots, w_{N}-2 W q_{N}\right]^{T}$. Note that the noise item in this model is different from that in the cluster-based sensor network. Therefore, it can be declared that the new mixing model depends on the sensor network model.

Remark 1: In the foregoing discussion, it is assumed that the transmissions are always successful. However, bit errors will occur due to noise corruption and channel distortion in harsh environments. This would affect the performance of source extraction in sensor networks. The focus of this paper is, however, on the effect of quantization of sensor observations, and the effects of noise and distortion will be addressed in future publications.

\section{RESULTS}

The performance of source extraction is illustrated by extensive simulations in this section. Two chaotic signals are used as sources, one being generated by a logistic map and the other by Chebyshev map, i.e., $s_{1}(t)=1-2 s_{1}^{2}(t-1), s_{2}(t)=$ $\cos \left(4 \arccos \left(s_{2}(t-1)\right)\right)$. The mixing coefficients are randomly generated from the standard normal distribution. The ambient noise levels [signal-to-noise ratio (SNR)] and the number of quantization bits are assumed to be identical for all sensors in the network. The correlation coefficient criterion [15] is adopted to evaluate the performance. The correlation coefficient of the source and the locally extracted signal is denoted by $\zeta^{e}$ and the correlation coefficient of the source and the globally extracted signal is denoted by $\zeta^{r}$. Computer simulations are run independently for $\beta$ times. In our study, we choose $\beta=1000$.

\section{A. Cluster-Based Sensor Network}

If $\zeta^{e} \geq 0.9$ and $\zeta^{r} \geq 0.8$, it is confirmed that a source is successfully extracted. This criterion stems from our observation that if $\zeta^{e} \geq 0.9$ and $\zeta^{r} \geq 0.8$, the globally extracted signal is sufficiently close to the source. The total number of successful extraction is evaluated. A new indicator called success rate is 

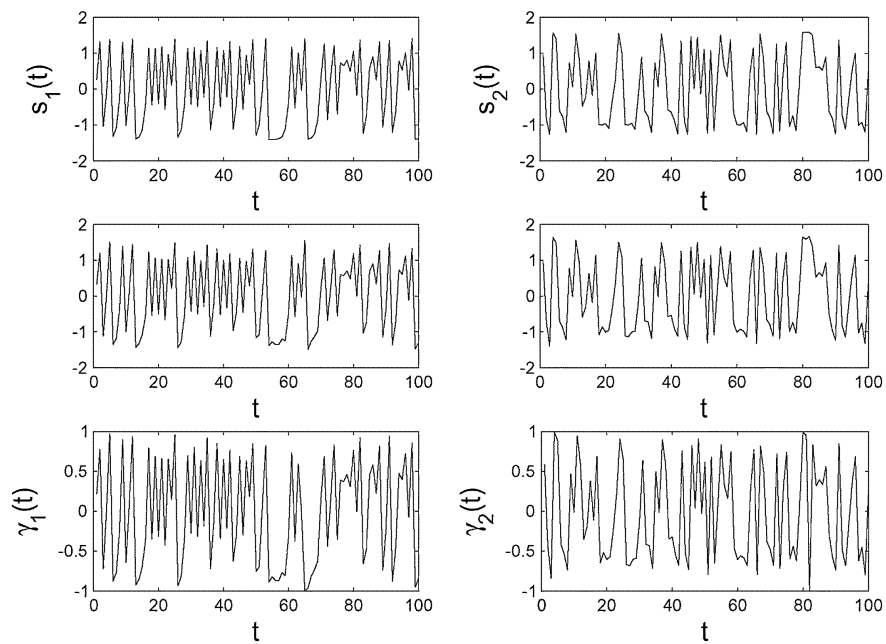

Fig. 3. The sources (first row), locally extracted signals (second row) and globally extracted signals (third row).

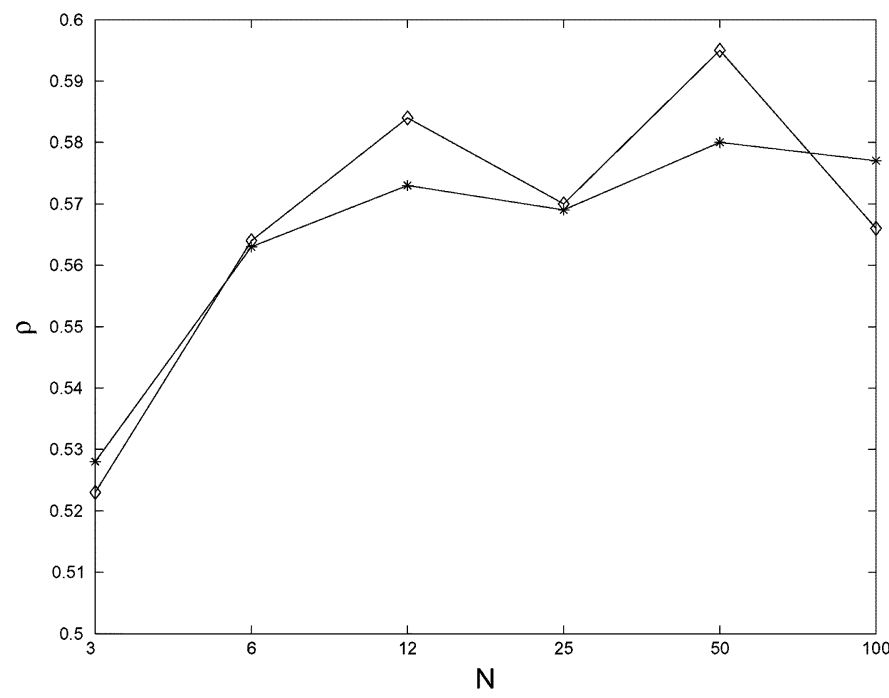

Fig. 4. Success rate $\rho$ versus the number of sensors $N$ with $M_{i}=4$ in the cluster-based sensor network. Solid line with $*$ corresponds to the proposed scheme and line with $\diamond$ corresponds to the benchmarking case.

defined as the ratio of the total number of successful extraction to $\beta$ and is denoted by $\rho$.

i) The parameters are set as $N=30, M_{i}=4, L=100$ and $\mathrm{SNR}=20 \mathrm{~dB}$. The sources are plotted in the first row of Fig. 3 , the locally extracted signals $\hat{s}_{1}$ and $\hat{s}_{2}$ in the second row, and the globally extracted signals $\gamma_{1}$ and $\gamma_{2}$ in the third row. Resemblance of the locally extracted signals and the globally extracted signals with the sources are clearly evident from Fig. 3.

ii) With $M_{i}=4$ and $\mathrm{SNR}=20 \mathrm{~dB}$, the success rate $\rho$ versus $N$ is shown in Fig. 4. It can be seen from Fig. 4 that $\rho$ generally increases with $N$. But when $N>12$, the performance gains very little. An intuitive explanation of this result is that more sensors provide more information about the sources. However, excessive information by no means contributes to the performance.

iii) With $N=12$ and $\mathrm{SNR}=20 \mathrm{~dB}$, the success rate $\rho$ versus $M_{i}$ is shown in Fig. 5. It is observed from Fig. 5 that $\rho$

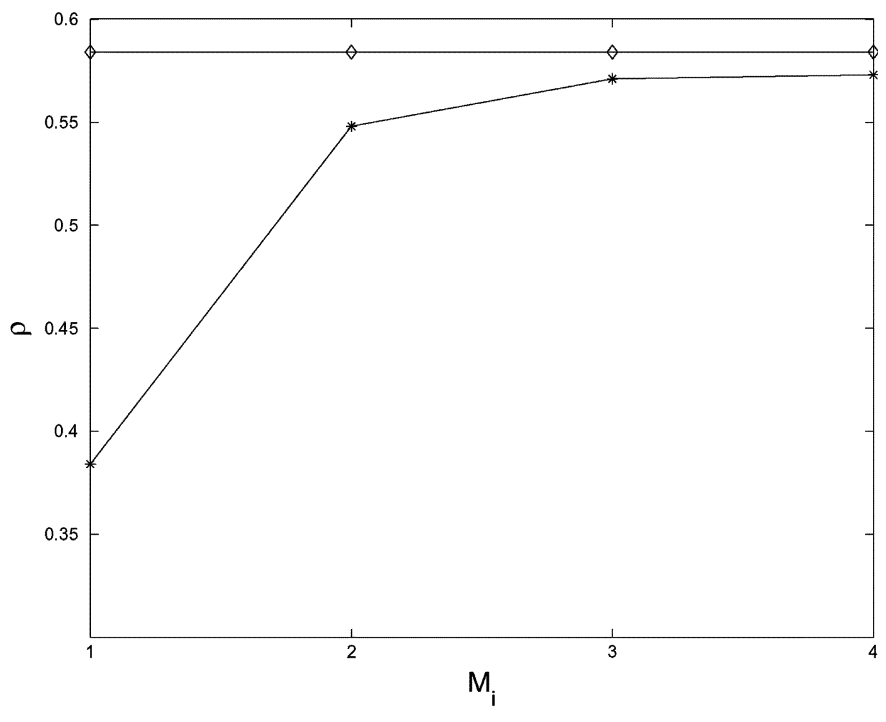

Fig. 5. Success rate $\rho$ versus the number of quantization bits $M_{i}$ in the clusterbased sensor network. Solid line with $*$ corresponds to the proposed scheme and line with $\diamond$ corresponds to the benchmarking case.

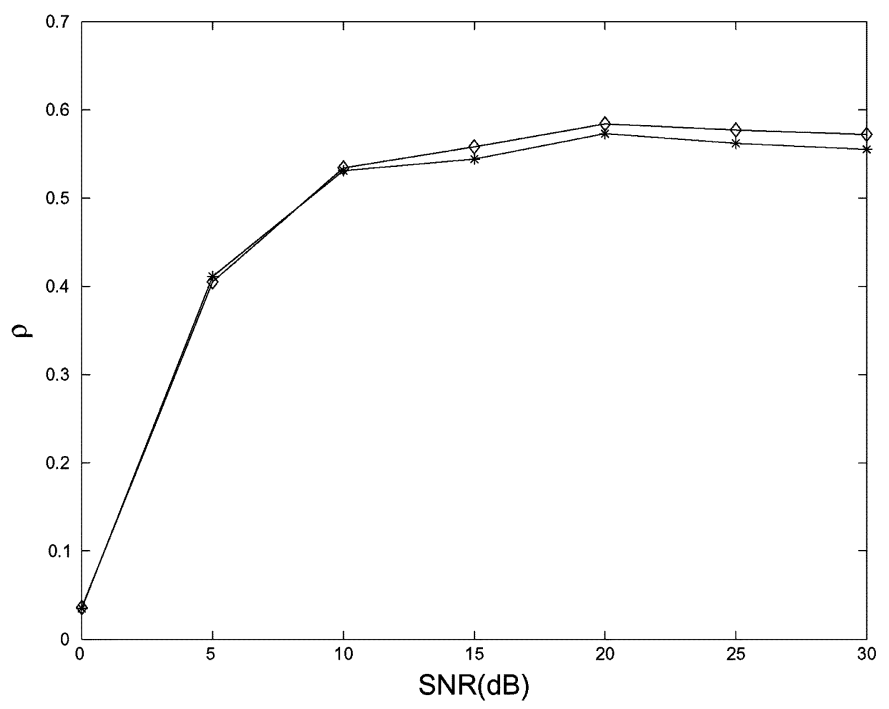

Fig. 6. Success rate $\rho$ versus SNR in the cluster-based sensor network. Solid line with $*$ corresponds to the proposed scheme and line with $\diamond$ corresponds to the benchmarking case.

increases with $M_{i}$. When $M_{i}=4$, the result is comparable to that of the benchmarking case. It also implies that when $M_{i}<4$, the bit representation of the original mixtures is not sufficient.

iv) With $N=12$ and $M_{i}=4$, the success rate $\rho$ versus SNR is shown in Fig. 6. As expected, $\rho$ generally increases with SNR. But when SNR $\geq 20 \mathrm{~dB}$, little further improvement can be observed. The reason behind this observation is that when SNR is high, the effect of noise is dominated by that of mixing.

v) The results of the benchmarking case are plotted in Figs. 4-6 for comparison. It is seen from Figs. 4-6 that the performance in this case is comparable to that of the benchmarking case.

vi) Let us take the most stringent bandwidth constraint into account, i.e., $M_{i}=1$. With $\mathrm{SNR}=20 \mathrm{~dB}$, the success 


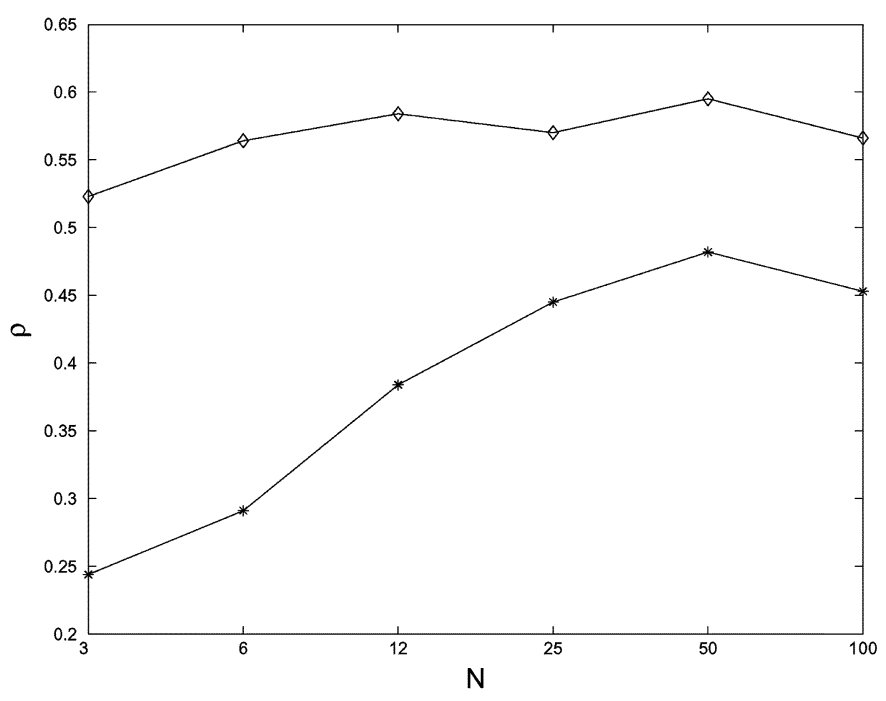

Fig. 7. Success rate $\rho$ versus the number of sensors $N$ with $M_{i}=1$ in the cluster-based sensor network. Solid line with $*$ corresponds to the proposed scheme and line with $\diamond$ corresponds to the benchmarking case.
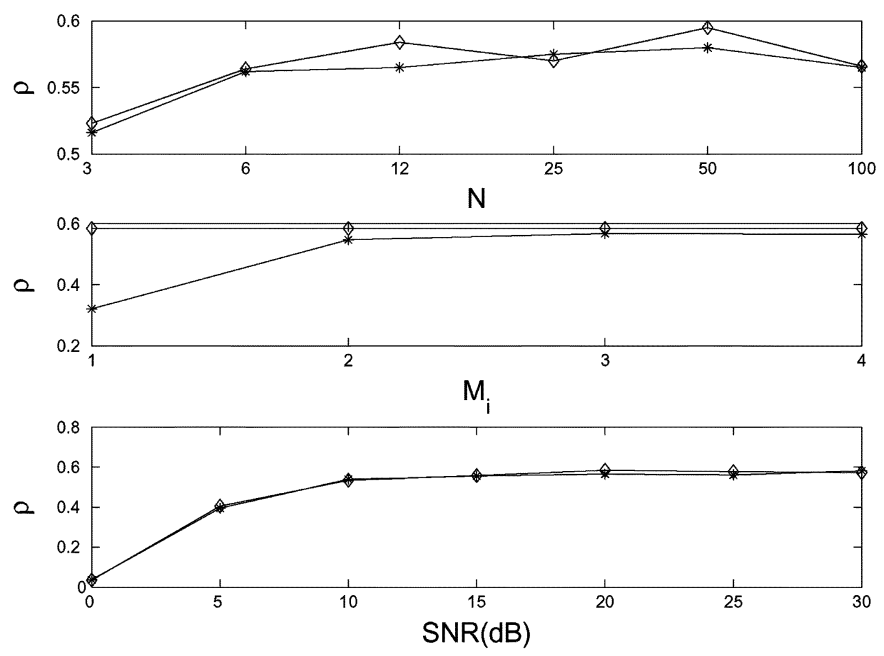

Fig. 8. Success rate $\rho$ versus $N, M_{i}$ and SNR in the cluster-free sensor network. Solid line with $*$ corresponds to the proposed scheme and line with $\diamond$ corresponds to the benchmarking case.

rate $\rho$ versus $N$ is shown in Fig. 7. It is apparent that even with 1-bit quantization, the sources can be extracted. The performance gap shrinks as $N$ increases and becomes stable when $N$ reaches 25 . This result is common in the studies of distributed estimation problem. For example, in [2]-[5], the performance achieved by the proposed estimator is inferior to that by the benchmarking estimator.

\section{B. Cluster-Free Sensor Network}

Only global extraction is involved in this case, so the success criterion is reduced to $\zeta^{r} \geq 0.9$. The success rate $\rho$ versus $N, M_{i}$ and SNR are shown in Fig. 8. The results of the benchmarking case are plotted in Fig. 8 for comparison. It can be seen from Fig. 8 that the performance in this case is also comparable to that of the benchmarking case and the trends of $\rho$ versus $N, M_{i}$, and SNR are similar to those in the cluster-based sensor network.

\section{CONCLUSION}

The problem of source extraction in bandwidth constrained wireless sensor networks is addressed. Simple schemes have been proposed for extracting multiple sources in a sensing field. Simulation results show that the proposed schemes can extract the sources effectively in both cluster-based and cluster-free sensor networks. The performance is comparable to that of the benchmarking case. Generally, if the sensor observations are distorted, the performance is not as good as that of the benchmarking case (this claim can be found in [5] and other references). It should be noted that if $L$ is sufficiently large and/or $\boldsymbol{w}$ is well initiated, the performance would improve.

When dealing with the source extraction problem in sensor networks, the interaction of signal processing and networking is potential to improve the performance. As stated in Section III-B, different sensor network models correspond to different mixing models. Hence, different algorithms may be devised for them. By now, recovering the sources from a noisy mixing model is still a difficult problem in signal processing. It is expected that the technical improvement in this aspect would accelerate the success of source extraction in sensor networks.

\section{REFERENCES}

[1] I. F. Akyildiz, W. Su, Y. Sankarasubramaniam, and E. Cayirci, "A survey on sensor networks," IEEE Commun. Mag., pp. 102-114, Aug. 2002.

[2] Z. Luo, "Universal decentralized estimation in a bandwidth constrained sensor network," IEEE Trans. Inf. Theory, vol. 51, no. 6, pp. 2210-2219, Jun. 2005.

[3] Z. Luo, "An isotropic universal decentralized estimation scheme for a bandwidth constrained ad hoc sensor network," IEEE J. Sel. Areas Commun., vol. 23, no. 4, pp. 735-744, Apr. 2005.

[4] J. Xiao and Z. Luo, "Decentralized estimation in an inhomogeneous sensing environment," IEEE Trans. Inf. Theory, vol. 51, no. 10, pp. 3564-3575, Oct. 2005.

[5] A. Ribeiro and G. B. Giannakis, "Bandwidth-constrained distributed estimation for wireless sensor networks-Part I: Gaussian case," IEEE Trans. Signal Process., vol. 54, no. 3, pp. 1131-1143, Mar. 2006.

[6] A. Ribeiro and G. B. Giannakis, "Bandwidth-constrained distributed estimation for wireless sensor networks-Part II: Unknown probability density function," IEEE Trans. Signal Process., vol. 54, no. 7, pp. 2784-2796, Jul. 2006.

[7] A. Ribeiro, G. B. Giannakis, and S. I. Roumeliotis, "SOI-KF: Distributed Kalman filtering with low cost communications using the sign of innovations," IEEE Trans. Signal Process., vol. 54, no. 12, pp. 4782-4795, Dec. 2006.

[8] T. Vercauteren and X. Wang, "Decentralized sigma-point information filters for target tracking in collaborative sensor networks," IEEE Trans. Signal Process., vol. 53, no. 8, pp. 2997-3009, Aug. 2005.

[9] A. Hyvärinen, J. Karhunen, and E. Oja, Independent Component Analysis.. New York: Wiley, 2001.

[10] A. Hyvärinen and E. Oja, "A fast fixed-point algorithm for independent component analysis," Neural Comput., vol. 9, pp. 1483-1492, 1997.

[11] A. Belouchrani, K. A. Meraim, J.-F. Cardoso, and E. Moulines, "A blind source separation technique using second-order statistics," IEEE Trans. Signal Process., vol. 45, no. 2, pp. 434-444, Feb. 1997.

[12] L. Tong, Y. Inouye, and R. Liu, "Waveform-preserving blind estimation of multiple independent sources," IEEE Trans. Signal Process., vol. 41, no. 7, pp. 2461-2470, Jul. 1993.

[13] J. G. Proakis, Digital Communications (3rd ed.).. New York: McGraw-Hill, 1995.

[14] N. S. Jayant, "Digital coding of speech waveforms: PCM, DPCM, and DM quantizers," Proc. IEEE, vol. 62, no. 5, pp. 611-633, May 1974.

[15] D. Farina, C. Févotte, C. Doncarli, and R. Merletti, "Blind separation of linear instantaneous mixtures of nonstationary surface myoelectric signals," IEEE Trans. Biomed. Eng., vol. 51, no. 9, pp. 1555-1567, Sep. 2004 